\title{
First high-resolution look at the quiet Sun with ALMA at $\mathbf{3} \mathbf{~ m m}$
}

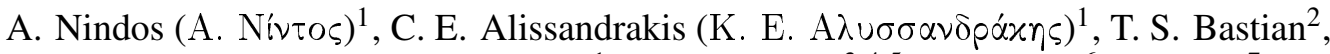 \\ S. Patsourakos $(\Sigma . ~ \Pi \alpha \tau \sigma o u p \alpha ́ x o \varsigma)^{1}$, B. De Pontieu ${ }^{3,4,5}$, H. Warren $^{6}$, T. Ayres ${ }^{7}$, \\ H. S. Hudson ${ }^{8,9}$, T. Shimizu ${ }^{10}$, J.-C. Vial ${ }^{11}$, S. Wedemeyer ${ }^{4}$, and V. Yurchyshyn ${ }^{12}$ \\ 1 Physics Department, University of Ioannina, Ioannina 45110, Greece \\ e-mail: anindos@cc.uoi.gr \\ 2 National Radio Astronomy Observatory, 520 Edgemont Road, Charlottesville, VA 22903, USA \\ 3 Lockheed Martin Solar and Astrophysics Laboratory, Palo Alto, CA 94304, USA \\ 4 Institute of Theoretical Astrophysics, University of Oslo, PO Box 1029, Blindern 0315, Oslo, Norway \\ 5 Rosseland Centre for Solar Physics, University of Oslo, PO Box 1029, Blindern 0315, Oslo, Norway \\ 6 Space Science Division, Naval Research Laboratory, Washington, DC 20375, USA \\ 7 Center for Astrophysics and Space Astronomy, University of Colorado, Boulder, CO 80309, USA \\ 8 School of Physics and Astronomy, University of Glasgow, G12 8QQ Glasgow, UK \\ 9 Space Sciences Laboratory, University of California, 7 Gauss Way, Berkeley, CA 94720-7450, USA \\ 10 Japan Aerospace Exploration Agency, 3-1-1 Yoshinodai, Chuo-ku, Sagamihara, Kanagawa 252-5210, Japan \\ 11 Institut d'Astrophysique Spatiale, UMR 8617, Université Paris 11, 91405 Orsay Cedex, France \\ 12 Big Bear Solar Observatory, New Jersey Institute of Technology, Big Bear City, CA 92314, USA
}

Received 21 August 2018 / Accepted 10 October 2018

\begin{abstract}
We present an overview of high-resolution quiet Sun observations, from disk center to the limb, obtained with the Atacama Large millimeter and sub-millimeter Array (ALMA) at $3 \mathrm{~mm}$. Seven quiet-Sun regions were observed at a resolution of up to $2.5^{\prime \prime}$ by $4.5^{\prime \prime}$. We produced both average and snapshot images by self-calibrating the ALMA visibilities and combining the interferometric images with full-disk solar images. The images show well the chromospheric network, which, based on the unique segregation method we used, is brighter than the average over the fields of view of the observed regions by $\sim 305 \mathrm{~K}$ while the intranetwork is less bright by $\sim 280 \mathrm{~K}$, with a slight decrease of the network/intranetwork contrast toward the limb. At $3 \mathrm{~mm}$ the network is very similar to the $1600 \AA$ Amages, with somewhat larger size. We detect, for the first time, spicular structures, rising up to $15^{\prime \prime}$ above the limb with a width down to the image resolution and brightness temperature of $\sim 1800 \mathrm{~K}$ above the local background. No trace of spicules, either in emission or absorption, is found on the disk. Our results highlight the potential of ALMA for the study of the quiet chromosphere.
\end{abstract}

Key words. Sun: radio radiation - Sun: chromosphere

\section{Introduction}

The chromosphere, which is the layer from which most of the solar radiation at millimeter $(\mathrm{mm})$ wavelengths is emitted, is still not fully understood. Recent significant progress in modeling its three-dimensional (3D) structure with sophisticated MHD models (see Hansteen et al. 2015; and references therein) has not succeeded in capturing a complete physical picture of the chromospheric fine structure and its dynamics (e.g., see De Pontieu et al. 2014; Tian et al. 2014). The main obstacle is the complexity of practically all available diagnostics, apart from those in the radio spectrum.

Observations of the radio continuum at $\mathrm{mm}$ wavelengths provide a unique chromospheric diagnostic. The quiet Sun mmwavelength emission mechanism is free-free and electrons are almost always in local thermodynamic equilibrium (for details, see Shibasaki et al. 2011; Wedemeyer et al. 2016).

There have been numerous observations of the mm- $\lambda$ emission of the Sun with single-dish telescopes; reviewed by Loukitcheva (2018). Most of them suffered from low spatial resolution and low sensitivity. These observations have been used to constrain empirical atmospheric models (see Shibasaki et al. 2011).
Interferometric observations of the Sun at mm wavelengths have been few and most of them were performed with a small number of telescopes, hence snapshot imaging was not possible (Labrum 1978; Belkora et al. 1992; White \& Kundu 1994). Probably the only exception in the pre-Atacama Large millimeter and sub-millimeter Array (ALMA) era is the set of observations reported by White et al. (2006) with the ten-element Berkeley-Illinois-Maryland Array (BIMA) at $85 \mathrm{GHz}$.

The availability of $\mathrm{mm}$ wavelength observations of the Sun with ALMA provides the potential to advance our knowledge of the chromosphere because of the instrument's unique capabilities in terms of spatial resolution and sensitivity. Extensive commissioning activities (see Shimojo et al. 2017; White et al. 2017) addressed the peculiarities of solar observation with ALMA and culminated in observations obtained in December 2015. These data led to several publications, reviewed by Loukitcheva (2018). In those, there is only one presentation of a quiet Sun image at $1 \mathrm{~mm}$ close to the limb (Shimojo et al. 2017).

In a previous article (Alissandrakis et al. 2017; hereafter Paper I), we used ALMA full-disk (FD) commissioning data to study the quiet Sun at low resolution; we computed the average center-to-limb variation (CLV) of the brightness temperature, $T_{\mathrm{b}}$, 
Table 1. Pointing and clean beam size of the ALMA $3 \mathrm{~mm}$ observations.

\begin{tabular}{ccccccccc}
\hline \hline Tgt & Aver UT & $\begin{array}{c}x \\
\left({ }^{\prime \prime}\right)\end{array}$ & $\begin{array}{c}y \\
\left({ }^{\prime \prime}\right)\end{array}$ & $R / R_{\odot}$ & $\mu$ & $\begin{array}{c}B_{\min } \\
\left({ }^{\prime \prime}\right)\end{array}$ & $\begin{array}{c}B_{\text {maj }} \\
\left({ }^{\prime \prime}\right)\end{array}$ & $\begin{array}{c}\text { PA } \\
\left({ }^{\circ}\right)\end{array}$ \\
\hline 1 & $15: 27: 35$ & -680 & -680 & 0.97 & 0.16 & 2.4 & 4.5 & -84 \\
2 & $16: 19: 51$ & -650 & -654 & 0.94 & 0.34 & 2.4 & 4.5 & -82 \\
3 & $17: 01: 38$ & -585 & -585 & 0.85 & 0.52 & 2.6 & 4.6 & -79 \\
4 & $18: 04: 06$ & -488 & -464 & 0.69 & 0.72 & 2.5 & 4.8 & -64 \\
5 & $18: 45: 54$ & -377 & -404 & 0.56 & 0.82 & 2.5 & 5.0 & -59 \\
6 & $19: 28: 03$ & -265 & -289 & 0.40 & 0.92 & 2.5 & 5.5 & -52 \\
7 & $20: 23: 29$ & 50 & 0 & 0.05 & 1.00 & 2.3 & 8.1 & -48 \\
\hline
\end{tabular}

and we derived the electron temperature, $T_{\mathrm{e}}$, as a function of optical depth. In this article we present high-resolution quiet-Sun observations that we obtained in March 2017; we present CLV measurements for the network and the intranetwork and discuss the properties of spicules beyond the limb.

\section{Observations and data reduction}

Seven regions (targets) were observed at $100 \mathrm{GHz}$ on March 16, 2017 , from the limb to the center of the disk, along a line at a position angle of $135^{\circ}$; each target was observed for $10 \mathrm{~min}$ with a cadence of $2 \mathrm{~s}$. In order to have the best coverage of low spatial frequencies, the observations were performed in the most compact ALMA configuration which included 40 twelve-meter antennas and 8 seven-meter antennas. Twenty-two FD images were obtained from two $12 \mathrm{~m}$ "total power" (TP) antennas (see White et al. 2017). The full width at half maximum (FWHM) of the single-dish beam was about $60^{\prime \prime}$ and each TP antenna scanned the full disk in $3 \mathrm{~min}$. Further information about the processing of the single-dish data is given in Appendix A. The weather conditions did not allow us to observe at $239 \mathrm{GHz}$, as we originally intended.

Table 1 gives the middle of the observing time intervals, the pointings in solar coordinates (selected to have a good sampling in $\mu$ ), the heliocentric distance, and $\mu$ for the seven targets, as well as the clean beam parameters (instrumental resolution). The resolution we achieved is close to the nominal resolution for the configuration we used which was $3.7^{\prime \prime} \times 2.5^{\prime \prime}$. The positions of the targets on a FD image are shown in Fig. A.1.

The visibilities were calibrated following the method described by Shimojo et al. (2017) and were provided to us by the Joint ALMA Observatory together with average CLEAN images. These images were not corrected for the primary beam response and no FD component had been added. Comparison of these images with simultaneous $1600 \AA$ images obtained by the Atmospheric Imaging Assembly (AIA) aboard Solar Dynamics Observatory (SDO) revealed that the ALMA pointing information provided in the visibilities was not accurate. Therefore the actual pointing was computed by cross-correlation of the ALMA with the AIA $1600 \AA$ images.

We imported the visibilities into the Astronomical Image Processing System (AIPS) and for each target we re-calculated CLEAN images synthesized from the entire observing period. We also calculated $2 \mathrm{~s}$ snapshot images. Both our average and snapshot CLEAN images were produced after four rounds of phase self-calibration, with solution intervals of $20 \mathrm{~min}, 1 \mathrm{~min}$, $2 \mathrm{~s}$, and $2 \mathrm{~s}$, and one round of amplitude self-calibration with a solution interval of $2 \mathrm{~s}$. The self-calibration, which is not included in the standard data pipelines, improved the image quality and removed the jitter that was apparent in the movies of snapshot images computed from the visibilities after the standard calibration procedure.
Subsequently, all images were imported into the Common Astronomy Software Applications (CASA) software, where they were combined with the processed FD image that is present in Fig. A.1. This procedure is necessary to (1) recover low spatial frequencies not accessible to the interferometric observations, and (2) obtain absolute brightness temperatures from the ALMA data. The combination was done using CASA's task feather and correcting for the fact that the flux of the derived images was higher than that of the FD image by $14 \%$, as noted in Paper I. Beyond the limb, we noticed in Target 1 an artificial $T_{\mathrm{b}}$ enhancement as high as $1000 \mathrm{~K}$ (see Fig. 2). This artifact may result from the incomplete sampling of the $\mathrm{u}-\mathrm{v}$ plane and/or nonoptimal weighting of the visibilities (see Shimojo et al. 2017 for details). The last step in the data reduction was the correction of all images for the primary beam response.

\section{Results}

A composite of all average ALMA images, each with a field of view (FOV) of $120^{\prime \prime}$ is presented in the top row of Fig. 1; the other rows show similar composites of the corresponding AIA data in the 1600-304 $\AA$ bands, convolved with the ALMA beam. In the course of this comparison we found a displacement towards the limb of the 304 images with respect to those at 1600 , indicating that the $304 \AA$ emission forms $4.8^{\prime \prime}$ ( $3 \mathrm{Mm}$ ) higher and this effect has been taken into account in Fig. 1 .

A first remark is that the quality of the ALMA images is very uniform over the FOV, which is twice the nominal FOV of $60^{\prime \prime}$, (defined as the FWHM of a single antenna), with some exceptions near the edge. The chromospheric network is clearly visible at $3 \mathrm{~mm}$, its morphology being very similar to that in the two AIA bands. There is also a very strong similarity between the ALMA images and the Interface Region Imaging Spectrograph (IRIS) slit-jaw images in three bands: 2796 (Mg II), 1440 (Si IV) and $1330 \AA$ (C II).

Using full-disk ALMA images, we noted in Paper I that the $239 \mathrm{GHz}$ network correlates slightly better with $1600 \AA$. Here with high resolution we find that the $100 \mathrm{GHz}$ images correlate equally well with the $1600 \AA$ and the $304 \AA$ images (crosscorrelation of $\sim 0.7$ ). Moreover, at $100 \mathrm{GHz}$ the structures are larger than at $1600 \AA$, but not as large as at $304 \AA$ (for targets $3-7$ the width of the autocorrelation function is $\sim 25 \%$ narrower at 1600 and $\sim 30 \%$ wider at $304 \AA$ ) suggesting that the radiation is formed in between. We note that at $\mathrm{cm}-\lambda$ the network correlates best with the $304 \AA$ band (Bogod et al. 2015).

Figure 2 shows the average brightness temperature, $T_{\mathrm{b}}$, derived from the ALMA interferometric images as a function of distance from disk center by performing azimuthal averaging. In the same figure we give the corresponding curves from the FD images of the present and the commissioning data (Fig. 4 of Paper I). We first note that the FD curves of this work and Paper I are remarkably similar, apart from some disk structures in the latter; indeed, during the present observations the Sun was much more quiet than in December 2015 (cf. Fig. 1 of Paper I with Fig. A.1). Apart from that, the high-resolution data of Fig. 2 show a moderate limb brightening, $\sim 5^{\prime \prime}$ wide, just beyond the photospheric limb.

In order to quantify the center-to-limb variation (CLV) of the network and cell interior we computed the average $T_{\mathrm{b}}$ of both for Targets $2-7$, as well as the average $T_{\mathrm{b}}$ and its root mean square (rms) value. We limited the FOV to $80^{\prime \prime}$ to minimize edge effects. We next fitted $T_{\mathrm{b}}(x, y)$ with a second-degree polynomial to avoid large-scale structure and we considered the average of the 


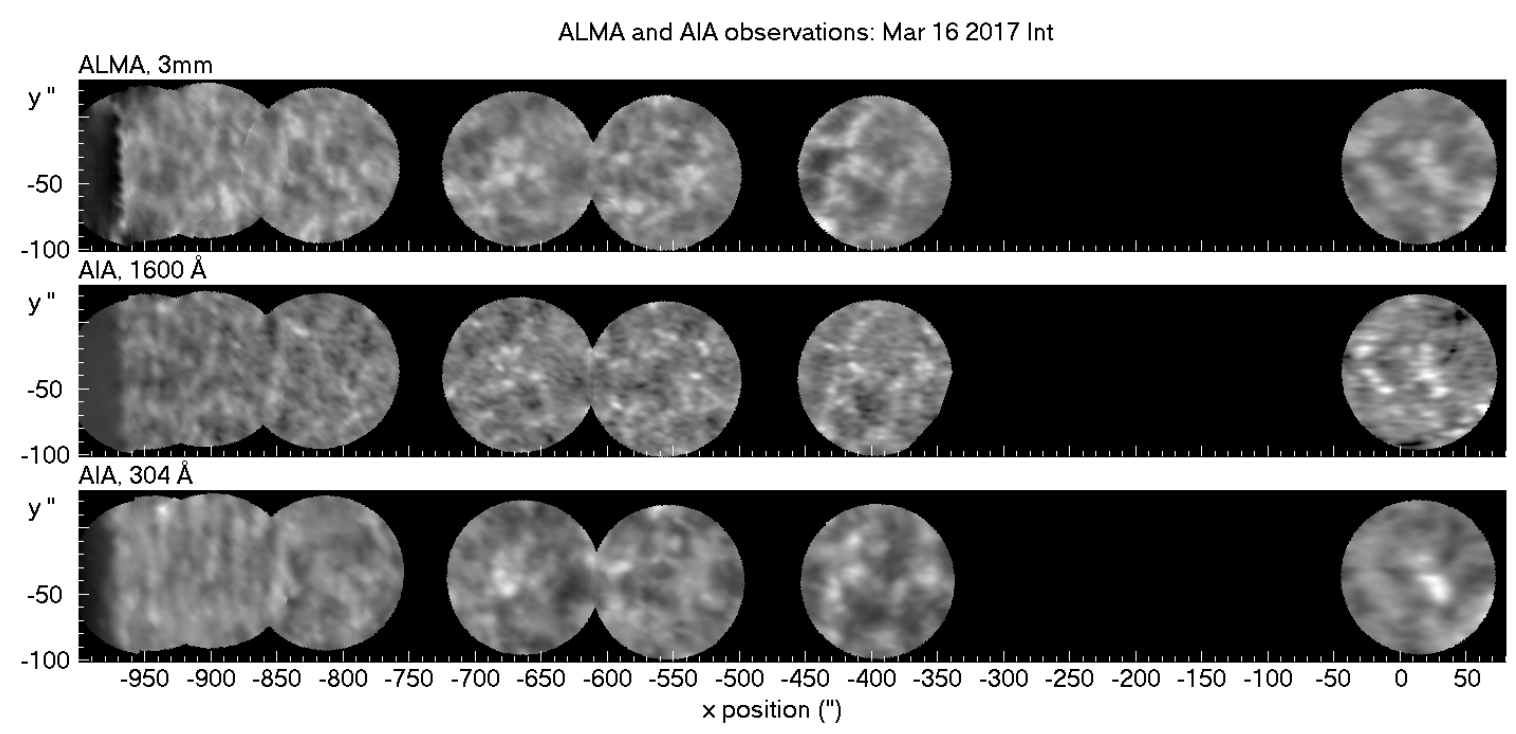

Fig. 1. Top row: composite of the seven $3 \mathrm{~mm}$ average ALMA images, corrected for solar rotation and reprojected at the position of their FOVs at 17:55:48 UT. Second and third rows: composites of AIA 1600 and $304 \AA$ images for the same time intervals and FOVs as the ALMA images, convolved with the ALMA beam. ALMA and $1600 \AA$ images are partly corrected for center-to-limb variation. The composites are oriented in the SW direction with respect to solar north, $x$ and $y$ are with respect to the disk center. The photospheric radius is $964.8^{\prime \prime}$.

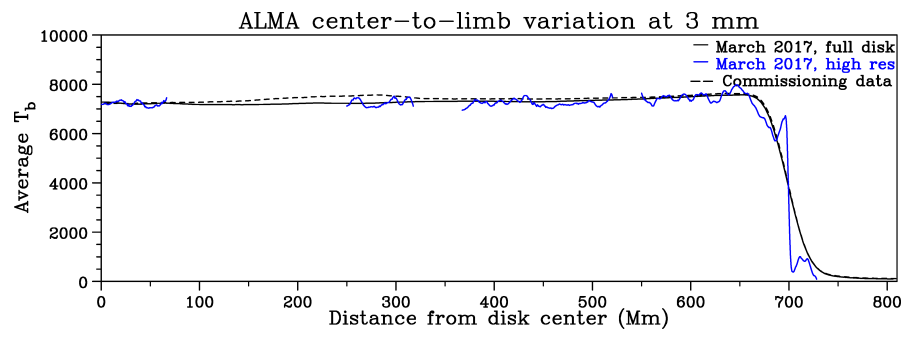

Fig. 2. Center-to-limb variation from the present and the commissioning full-disk data (full and dashed black curves) and from the interferometric average images with a FOV of $110^{\prime \prime}$ (blue curve).

Table 2. Network, cell interior average and $\mathrm{rms} T_{\mathrm{b}}$ in $\mathrm{K}$.

\begin{tabular}{ccccccccc}
\hline \hline$\mu$ & Net & Cell & Aver & rms & $\begin{array}{c}\text { Net/Cell } \\
\text { Ratio }\end{array}$ & $\begin{array}{c}\text { Net }- \\
\text { Cell }\end{array}$ & $\begin{array}{c}\text { Net }- \\
\text { Aver }\end{array}$ & $\begin{array}{c}\text { Cell }- \\
\text { Aver }\end{array}$ \\
\hline 0.34 & 7670 & 7120 & 7370 & 570 & 1.077 & 550 & 300 & -250 \\
0.52 & 7760 & 7150 & 7460 & 440 & 1.084 & 600 & 300 & -310 \\
0.72 & 7500 & 7030 & 7270 & 400 & 1.066 & 470 & 240 & -230 \\
0.82 & 7670 & 7030 & 7330 & 430 & 1.091 & 640 & 340 & -300 \\
0.92 & 7620 & 6950 & 7270 & 460 & 1.097 & 670 & 350 & -330 \\
1.00 & 7530 & 6940 & 7220 & 390 & 1.085 & 590 & 300 & -290 \\
\hline
\end{tabular}

values above the fit as representative of the network and the others as representative of the intranetwork. This method of separating network/internetwork is by no means unique, but leads to a reasonably good network/internetwork segregation (see insert in Fig. 3). The results are given in Table 2 and plotted in Fig. 3.

Both the network and the cell interior show limb brightening. Their difference, as well as the difference between the network and the average $T_{\mathrm{b}}$, decrease marginally towards the limb, while the difference between the cell interior and average difference shows a marginal increase. The network/cell ratio has an average value of 1.08 and decreases slightly towards the limb. On average, the network is $305 \pm 35 \mathrm{~K}$ above the average $T_{\mathrm{b}}$ and the intranetwork $285 \pm 30 \mathrm{~K}$ below. Our results are only slightly affected by the ALMA resolution; a check using AIA $1600 \AA$ data for Target 5 gave a $3.3 \%$ increase of the cell intensity and a $3.7 \%$ drop of the network intensity after convolution with the ALMA beam.

Structures beyond the limb are easily visible in Fig. 1. A closer look shows that they are spicules, or rather groups of spicules (Fig. 4), as evidenced from the comparison of ALMA with $\mathrm{H} \alpha$ images from the GONG network. The most prominent structure, on the left and marked by an arrow in Fig. 4, is almost parallel to the ALMA beam, thus facilitating its detection. It is visible from $\sim 2$ to $\sim 15^{\prime \prime}$ above the limb and is most prominent at $8.5^{\prime \prime}$, with $T_{\mathrm{b}}=2560 \mathrm{~K}, 1800 \mathrm{~K}$ above the local background and a width of $4^{\prime \prime}$. Lower down, $T_{\mathrm{b}}$ goes up to $6500 \mathrm{~K}$ and the width drops to $2.5^{\prime \prime}$, very close to the instrumental resolution of $2.4^{\prime \prime}$. Their lifetime is comparable to the 10 min duration of the Target 1 observations. Although the roots of some spicular structures in Fig. 4 are inside the limb, we found no evidence of spicules on the disk, either in emission or in absorption.

\section{Summary and discussion}

Although the weather conditions during our ALMA observations were far from ideal, we obtained good average and snapshot images through the tedious process of self-calibration, with a resolution very close to the theoretical $\left(2.4^{\prime \prime} \times 4.5^{\prime \prime}\right)$. The most serious problem with our final images is that we got emission beyond the limb of up to $1000 \mathrm{~K}$ ( $14 \%$ of the disk center value), the origin of which we could not identify. This excess emission occurs well beyond the region where the spicules are located and therefore its presence does not affect the brightness temperature measurements of the spicules. Apart from that, our images appear good over a circular FOV with a diameter of about $120^{\prime \prime}$, which is twice the nominal ALMA FOV at $100 \mathrm{GHz}$. The good quality of the images was judged by the fact that no significant artifacts appeared close to the edge of the FOV.

Our images show clearly the chromospheric network; morphologically they are close to AIA $1600 \AA$ and $304 \AA$. Individual ALMA features are broader than at $1600 \AA$, indicating that the $3 \mathrm{~mm}$ radiation on the disk is formed between the 1600 and the 304 levels; we found that the latter is formed $3 \mathrm{Mm}$ above the 1600 level. 

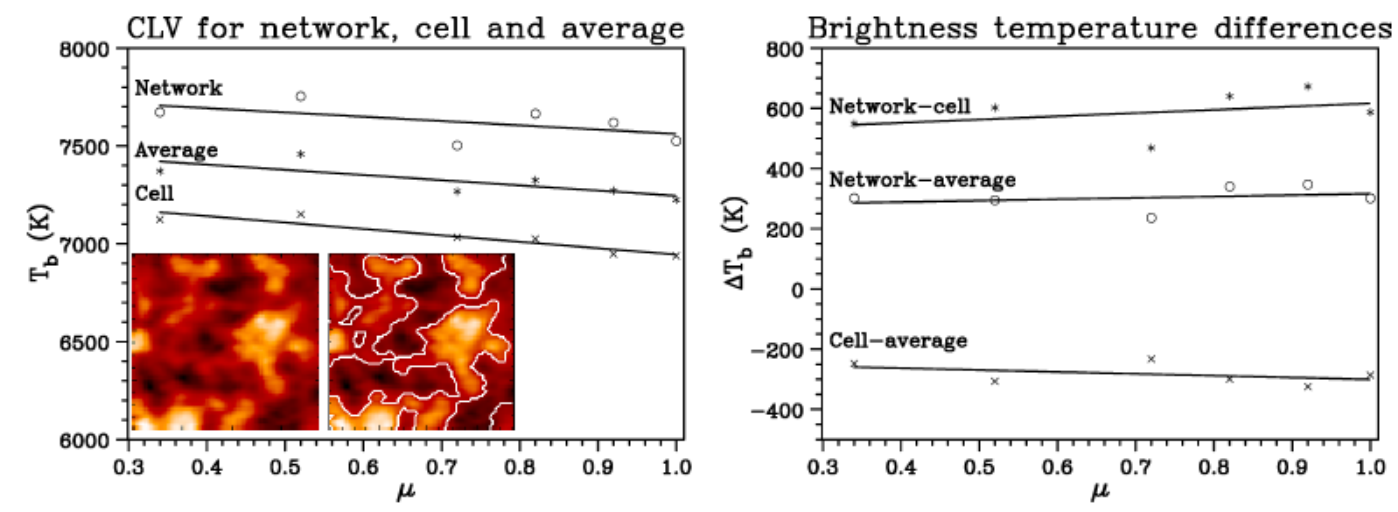

Fig. 3. $T_{\mathrm{b}}$ of the network, cell interior, and average (left panel); and their differences (right panel) as a function of $\mu$. The insert shows the inner $80^{\prime \prime} \times 80^{\prime \prime}$ of Target 5; contours show the network-cell boundaries.

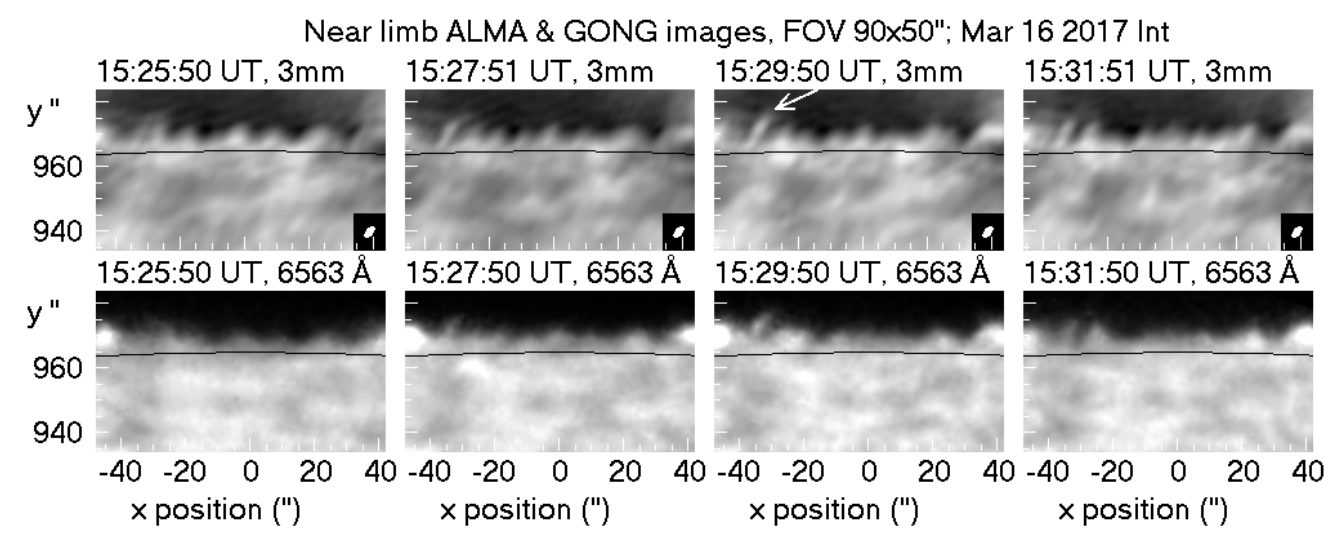

Fig. 4. Spicules seen in near-limb images at $3 \mathrm{~mm}$ (top panels) and $\mathrm{H} \alpha$ (bottom panels). All images have been rotated to make the limb parallel to the horizontal direction and corrected to reduce the sharp drop of intensity near the limb by subtracting $90 \%$ of the azimuthally averaged intensity. The black curve marks the photospheric limb and the inserts show the ALMA beam.

The CLV curve from the present FD data is remarkably similar to the one we derived from the commissioning data in Paper I, while the high-resolution data show a moderate and narrow limb brightening just above the photospheric limb. The network is brighter than the average by $\sim 305 \mathrm{~K}$ and the intranetwork is less bright by $\sim 280 \mathrm{~K}$, with a slight decrease of the network/intranetwork contrast towards the limb; this is crucial information for atmospheric modeling.

We report for the first time the detection of spicular structure beyond the limb, with a width down to our theoretical resolution, detectable up to $15^{\prime \prime}$ above the limb and with $T_{\mathrm{b}} \sim 1800 \mathrm{~K}$ above the local background. Interestingly, no trace of spicules appears in the $1 \mathrm{~mm}$ image in Shimojo et al. (2017). We see no trace of spicules on the disk, either in emission or in absorption, probably due to insufficient resolution or small optical thickness; further work is required to deduce whether their effect on the CLV of the brightness is small or, as is usually assumed (e.g., Lantos \& Kundu 1972; Selhorst et al. 2005), substantial.

In this article we present a broad overview of the first highresolution ALMA observations of the network at $3 \mathrm{~mm}$. In subsequent work we intend to exploit the CLV data for two-component atmospheric modeling, elaborate further on the larger size of the structures at $3 \mathrm{~mm}$ than at lower heights, compare with magnetograms, and study oscillations and transients and their effect on the corona. From the observational point of view, similar data at $239 \mathrm{GHz}$ and higher frequencies are highly desirable, as well as measurements of circular polarization and observations with more extended configurations and thus higher resolution.
Acknowledgements. This paper makes use of the following ALMA data: ADS/JAO.ALMA\#2016.1.00572.S. ALMA is a partnership of ESO (representing its member states), NSF (USA) and NINS (Japan), together with NRC (Canada) and NSC and ASIAA (Taiwan), and KASI (Republic of Korea), in cooperation with the Republic of Chile. The Joint ALMA Observatory is operated by ESO, AUI/NRAO and NAOJ. IRIS is a NASA small explorer mission developed and operated by LMSAL with mission operations executed at NASA Ames Research center and major contributions to downlink communications funded by ESA and the Norwegian Space Centre.

\section{References}

Alissandrakis, C. E., Patsourakos, S., Nindos, A., \& Bastian, T. S. 2017, A\&A, 605, A78 (Paper I)

Belkora, L., Hurford, G. J., Gary, D. E., et al. 1992, ApJ, 400, 692

Bogod, V. M., Alissandrakis, C. E., Kaltman, T. I., \& Tokhchukova, S. K. 2015, Sol. Phys., 290, 7

De Pontieu, B., Rouppe van der Voort, L., McIntosh, S. W., et al. 2014, Science, 346,1255732

Hansteen, V., Carlsson, M., \& Gudiksen, B. 2015, ASP Conf. Ser., 498, 141 Labrum, N. R. 1978, PASA, 3, 256

Lantos, P., \& Kundu, M. R. 1972, A\&A, 21, 119

Loukitcheva, M. 2018, Adv. Space Res., in press, DOI: 10.1016/j.asr.2018.08.030

Loukitcheva, M., Solanki, S. K., Carlsson, M., et al. 2004, A\&A, 419, 747

Selhorst, C. L., Silva, A. V. R., \& Costa, J. E. R. 2005, A\&A, 433, 365

Shibasaki, K., Alissandrakis, C. E., \& Pohjolainen, S. 2011, Sol. Phys., 273, 309

Shimojo, M., Bastian, T. S., Hales, A. S., et al. 2017, Sol. Phys., 292, 87

Tian, H., DeLuca, E. E., Cranmer, S. R., et al. 2014, Science, 346, 1255711

Wedemeyer, S., Bastian, T., Brajša, R., et al. 2016, Space Sci. Rev., 200, 1

White, S. M., \& Kundu, M. R. 1994, IAU Symp., 154, 167

White, S. M., Loukitcheva, M., \& Solanki, S. K. 2006, A\&A, 456, 697

White, S. M., Iwai, K., Phillips, N. M., et al. 2017, Sol. Phys., 292, 88 


\section{Appendix A: Full-disk images}

During our ALMA observation, 22 FD images were obtained with antennas 0 and 1. Figure A.1 (left) shows one of them, partly corrected for center-to-limb variation. As in our previous work (cf. Fig. 1 of Paper I), the FD image shows spokewheel-like irregularities near the limb, which could influence the "feathering" procedure for near-limb Target 1 . We reduced these features by low pass azimuthal filtering of the region near the limb with a $120^{\prime \prime}$ wide Gaussian. We also corrected the FD image for the slight ellipticity of the solar disk, also reported in Paper I. The corrected image is shown at the right of Fig. A.1; the dark disks mark the position of the seven targets and their diameter is equal to a FOV of $120^{\prime \prime}$.

In order to facilitate comparison of future modeling studies of our data with those presented in Paper I, we scaled the FD image to match the quiet Sun brightness temperature at disk center reported in Paper I which was $7250 \mathrm{~K}$. This value is only $50 \mathrm{~K}$ lower (about $0.7 \%$ ) than the value recommended by White et al. (2017), that is, 2 and 3.4 times smaller than the relevant uncertainties reported in White et al. (2017) and Paper I, respectively.

We found that the addition of the large-scale spatial components did not change the image structure appreciably, except for

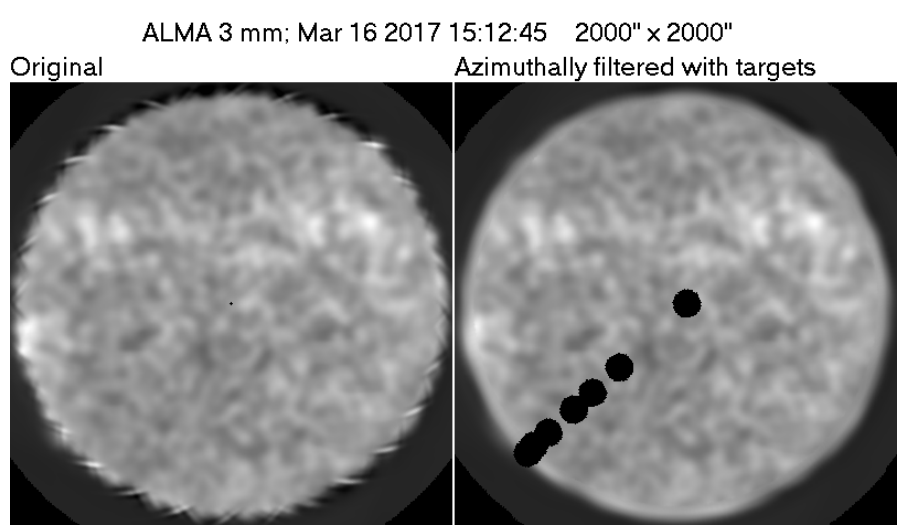

Fig. A.1. Full-disk ALMA images: Left panel: original. Right panel: after azimuthal filtering, correction of the limb ellipticity and with the seven targets added as black disks of $120^{\prime \prime}$ diameter. In both images $90 \%$ of the azimuthally averaged intensity has been subtracted to enhance the visibility of disk features.

the near-limb Target 1 . For this target we took particular care to match the FD and interferometric limbs, by matching the inflection points of their CLV curves. 